\title{
Research on Influence Factors of Quantitative Perception of Internet Word of Mouth Based on Online Reviews
}

\section{Jinhai Li ${ }^{\mathbf{1}}$}

Taizhou University

Taizhou 225300, China

Email: $1 j h-h k @ 163 . c o m$

\section{Yunlei Ma}

Taizhou University

Taizhou 225300, China

Email: 562547791 @qq. com

\section{Huisheng Zhu}

Taizhou University

Taizhou 225300, China

Email: 2848961504 @qq. com

\section{Youshi He}

School of Management, Jiangsu University

Zhenjiang 212013, China

Email: 1603998118 @qq. com

In order to accurately measure the value of internet word of mouth (iwom). Based on each attribute of online reviews, which are the major form of iwom, we proposes the influence factors of iwom, include the quantifiable indicators: the number of online reviews, the rating, the proportion of negative reviews, the price of product, and the unquantifiable indicators which can control of the false online reviews and express consumer emotion: the feature words of product attributes, the emotional intensity of reviews. Then we build the model of influence factors of iwom perception. And we take the influence of endogenous into account. Before and after the control of the endogenous influence, we find that the relationship between influence factors and iwom has changed by using cross section analysis and first order difference analysis.

1 This study is supported by the Scientific Research Foundation of Taizhou University (QD2016036), the National Social Science Foundation of China (16BGL088), Science and Technology Support Project of Taizhou (TS201515)and the Natural Science Foundation of Jiangsu Province (BK20141307). 


\section{Introduction}

The traditional word of mouth is an informal communication of information such as brand, product and service among consumers which is not for the purpose of commercial marketing. Its influence is subjected to the restrictions of the consumers' range of social greatly. And as time goes on, its influence will diminish quickly. Recently, more consumers release the product reviews on the internet with the popularity of iwom [1].

Based on the characteristics of low cost and two-way communication of internet, iwom overcomes defect of traditional wom which can only spread within the limited social boundary and weaken fast with the increasing of the time and distance. And because of the anonymity and timeliness of the internet, consumers can spread the information to anyone at any time. It makes the influence of iwom to the spread of the geometric level.

Consumers can post perception of product in the form of review on the internet freely. It can quickly get all kinds of positive, negative or neutral reviews from consumers together, to form iwom. Potential customers will acquire the perception of iwom of product through browsing these reviews. It will affect their purchase decisions, thus influence the sales of the products. Many studies have shown that the influence of negative iwom is more prominent than the influence of positive iwom[2]. Therefore, always understand the change of iwom is not only the compulsory course before consumers to buy the product, but also the important reference for enterprises to improve product and service.

Online reviews are a main embodiment of iwom. It is a customer oriented information and different from the information which provided by enterprise. It is also a supplement to the personalized recommendation which includes the product description, expert reviews and automatically generated information by e-commerce system. Due to the high reliability and strong experience of online reviews, it is often considered as more reference value than the information which provided by enterprise. Online reviews as quantifiable text information include structure characteristics such as number, length and rating. But due to lack of effective regulation and control means, the related interest groups use the convenience of internet platform to release false online reviews which are good for their own products but against their competitors' products. It reduces the credibility of online reviews. So if we measure iwom of products only by the number of online reviews and rating, we cannot quantify the value of iwom accurately.

The paper introduces the quantifiable and unquantifiable indicators of iwom on the basis of combining previous qualitative research of iwom.

\section{The Determination Of Quantitative Indicators Of Iwom}

\subsection{The quantifiable indicators}

(1) The number of online reviews

By summarizing the study of iwom, we find that the key objective characteristics of online reviews (number and rating) have influence on iwom perception [3]. The number of online reviews decides the number of consumers who participate in discussing about product. The more reviews 
mean more consumers attention to the product [4]. This conclusion is further validation by Duan through studying the relationship of movie reviews and box office in the Yahoo website [5]. It shows that the number of online reviews is one of the important factors in the process of quantitative perception of iwom. Therefore, we hypothesize H1:

H1: The number of online reviews has a positive influence on quantitative perception of iwom.

(2) The rating of online reviews

And the rating of reviews is a direct response to the product by consumers. It is generally believed that the higher rating the better evaluation by consumers. Then potential consumers will enhance recognition of product iwom. However, through the existing research we find that the conclusion about the influence on iwom perception from rating is inconsistent by scholars. Some scholars found that the consumers who contact with the positive reviews bought products on the frequency of 2 times to other consumers [6], while Liu (2006) put forward a question through empirical research based on movie reviews from Yahoo. His result showed that the rate of online reviews had no direct influence on film [7].

Although he conclusion about the influence on iwom from the rating of online reviews is inconsistent, its importance is no doubt. The paper assumes that rating will influence quantitative perception of iwom. And its influence will be further verification through the model. Therefore, we hypothesize $\mathrm{H} 2$ :

$\mathrm{H} 2$ : The rating of online reviews has a certain positive influence on quantitative perception of iwom.

(3) The proportion of negative online reviews

Negative online reviews can lead to negative perception of iwom about products [8]. And the influence on reducing the product sales by negative reviews is more significant than the influence on increasing the product sales by positive reviews [3].

The paper regards the proportion of negative online reviews as an important supplement of measuring negative iwom perception based on previous research conclusion in order to avoid possible deviation by using the rating of reviews simply. Therefore, we hypothesize H3:

H3: The proportion of negative reviews has a negative influence on quantitative perception of iwom.

(4) The price of product

The price has two sides' dual effects of iwom. A low price is a prerequisite for more consumers contact with product. The low-priced products are more easily accepted by consumers relative to the high-priced. But the price is also an embodiment of the quality of the product. Higher price means higher quality assurance for the consumers. It will lead to an improvement in product iwom in a certain extent. However, too high or too low prices will add to perceived risk of product [9]. Therefore, we hypothesize H4:

H4: The price of product has certain adjustment of quantitative perception of iwom.

Aim at the question of the influence degree from the rating of online reviews and the price of product to quantitative perception of iwom is uncertain, mainly because at present the most 
researches regard attribute of online reviews as exogenous variables of influence iwomand ignore the endogenous problem which maybe generate from the influence factors model. It leads to overvalue the effect of online reviews. Therefore, the paper explains the influence from endogenous problem to quantitative perception of iwom through cross section analysis and first order difference analysis respectively.

\subsection{Control influence of endogenous}

The existence of endogenous leads to the estimation results of independent variable coefficient produce errors in the econometric model [10]. The important reason of producing endogenous is missing important relevant variables in the model. The endogenous is an important problem which affects the research conclusion in the relationship analysis between attribute of online reviews and quantitative perception of iwom. Because the intrinsic properties such as the brand effect and the quality of product can influence online reviews and quantitative perception of iwom of product by consumers at the same time, the endogenous problem is produced that leads to overvalue the effect of online reviews. Therefore, we hypothesize H5 and H6:

H5: When the influence of endogenous is controlled, the influence degree from the rating of online reviews to quantitative perception of iwom will be weakened.

H6: When the influence of endogenous is controlled, the influence degree from the price of product to quantitative perception of iwom will be weakened.

\subsection{The unquantifiable indicators}

The key objective characteristics such as the number and rating of online reviews can be manipulated by wildcatter through a lot of frauds in the era of the open internet. In order to comprehensively measure the product iwom, the paper introduces the unquantifiable indicators of iwom for avoiding the error of the quantitative indicators caused by vicious competition [11].

(1) The feature words of product attributes

The feature words of product attributes are the objective description of the product itself and its services. The number of feature words which mined from online reviews relates to the fit of product that reviews described. The more the review has feature words, the higher relevance between review and product, and then it has more useful to quantitative perception of iwom of product. Therefore, we hypothesize H7:

H7: The feature words of product attributes have a positive influence on quantitative perception of iwom.

(2) The emotional intensity of reviews

When consumers release online reviews, they will make emotional evaluation based on their using experience and subjective feelings. The emotional intensity of reviews will reflect the emotional tendencies of consumers for the product. The positive emotion means recognition of products. It can develop the iwom of product. Therefore, we hypothesize H8:

H8: The emotional intensity of reviews has a positive influence on quantitative perception of iwom. 


\subsection{The theoretical model}

Based on previous research conclusion and the theoretical assumptions, the paper mainly analyzes the influence on value perception of iwom by the quantifiable indicators and unquantifiable indicators. The model of influence factors of iwom perception is shown as in figure 1.

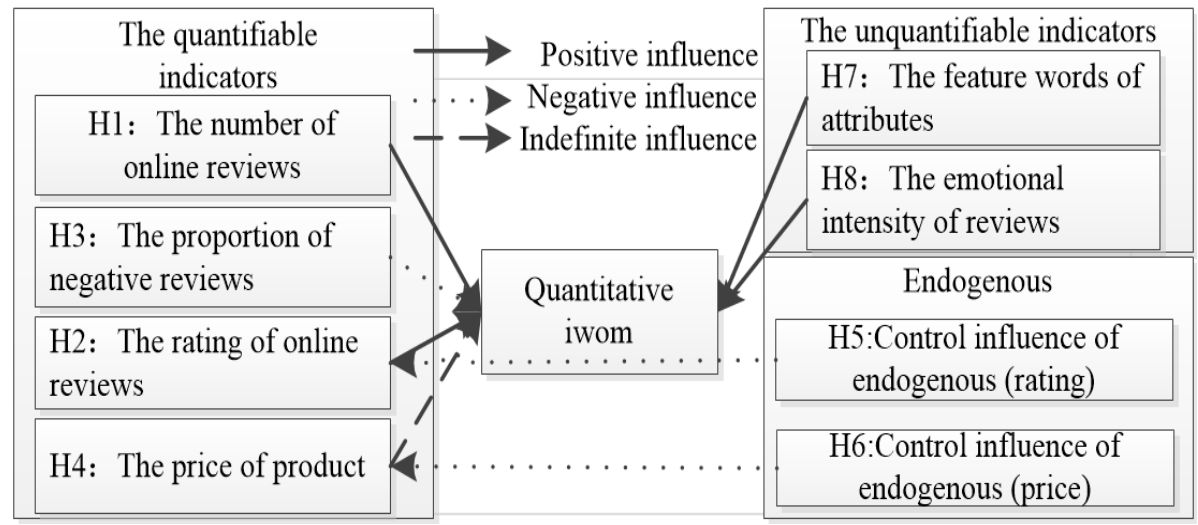

Figure 1: The model of influence factors of iwom

\section{The Influence From Online Reviews To Quantitative Perception Of Iwom}

\subsection{Data sources and variables analysis}

Online reviews that studied in the paper are text including product quality, service quality and rating which consumers release in $\mathrm{B} 2 \mathrm{C}$ e-commerce. And the amazon is one of the main online shopping platforms. The paper chooses mobile phones as experimental research object which release during March 1, 2015 to June 1, 2015 from amazon. The deadline of grabbing online reviews is July 1, 2015. This is to ensure that the number of online reviews of new released mobile phone can meet certain requirement. The paper regards the number of online reviews greater than 100 as the restrictive conditions to filter the grabbing online reviews, and we get 185 effective products after removing the phones which do not meet the requirements of number.

Considering the endogenous problems including the quality of product, brand effect and so on possible to cause the estimate error of each attribute of online reviews, the paper introduces the brands rank, the number of competitors, the magnitude of reduction and the release time of product as the control variables into model.

In order to comprehensive analyze the influence from each attribute of online reviews to quantitative perception of iwom, the paper establishes two independent linear models named model 1 and model 2 between quantitative perception of iwom and each influence factors based on the results of the above analysis. In order to avoid the effects of the heteroscedasticity and skewness, some variables in the model take the form of natural logarithm:sale

Model 1:

$$
\ln \left(\text { sale }_{i}\right)=\alpha+\alpha_{1} \ln (\text { rank })+\alpha_{2} \ln (\text { competitor })+\alpha_{3} \text { reduction }+\alpha_{4} \ln (\text { date })
$$


Where the dependent variable of quantitative perception of iwom (sale) is expressed by the logarithm value of sales rank of the mobile phone. rank is expressed by the brands rank, competitor is expressed by the number of competitors, reduction is expressed by the magnitude of reduction, and date is expressed by the release time of product. $\alpha$ is the constant term, $\alpha_{1}, \alpha_{2}, \alpha_{3}$ and $\alpha_{4}$ are the coefficient of rank, competitor, reduction and date respectively.

Model 2:

$$
\begin{aligned}
\ln \left(\text { sale }_{i}\right)= & \alpha+\alpha_{1} \ln (\text { rank })+\alpha_{2} \ln (\text { competitor })+\alpha_{3} \text { reduction }+\alpha_{4} \ln (\text { date }) \\
+ & \beta_{1} \ln (\text { number })+\beta_{2} \frac{1}{\text { number }_{i}}+\beta_{3} \text { negp }+\beta_{4} \ln (\text { price }) \\
& +\beta_{5} \frac{1}{\text { number }_{i}} \sum \text { feature }+\beta_{6} \frac{1}{\text { number }_{i}} \sum \text { emotion }
\end{aligned}
$$

Where the indepedentvariable of number is expressed by the actual statistical number of reviews, rate is expressed by the rating ofonlinereviews, negp is expressed by the number of rating with 1 accounted for the proportion of the total number of reviews, price is expressed by the average price during the time of product release to the time of grabbing online reviews, feature is expressed by the number of feature words of product attributes which extract from online reviews, emotion is expressed by the level of emotional intensity of reviews. number ${ }_{i}$ is the number of online reviews belongs to ith mobile phone. $\beta_{1}, \beta_{2}, \beta_{3}, \beta_{4}, \beta_{5}$ and $\beta_{6}$ are the coefficient of number, rate, negp, price, feature and emotion respectively. The function of model 1 is observation whether the goodness-of-fit of the model has obvious ascension when the variables of online reviews added, in order to illustrate that whether each attribute of online reviews have important influence on iwom perception.

\subsection{The cross-section analysis}

In the model 2 the paper needs to analyze the influence from each attribute of online reviews to the iwom perception, which is the plus-minus sign and significant of $\beta_{1} \sim \beta_{6}$. Based on the above assumptions, the variables of number, rate, feature and emotion have the positive influence on iwom perception, negp has a negative influence on iwom perception, and price has certain adjustment of iwom perception. Because the smaller the sales ranking is, the higher iwom perception is. Therefore, we can speculate that $\beta_{1}, \beta_{2}, \beta_{5}, \beta_{6}<0, \beta_{3}>0$ and $\beta_{4}$ is indefinite. Because we add 4 control variables to the model 1 and 2 , it may cause the multicollinearity problem. Therefore, in order to avoid potential multicollinearity problem, the corresponding variables are carried on the centralized processing. And we calculate the variance inflation factor, all $\mathrm{VIF}<5$. It means that the multicollinearity of the model is controlled within the acceptable range. The results of the crosssection regression analysis of model 1 and 2 are shown as table 1. 


\begin{tabular}{|l|l|l|}
\hline Variables & Model 1 & Model 2 \\
\hline rank & $0.425(0.251)$ & $0.384^{* *}(0.233)$ \\
\hline competitor & $0.241(0.176)$ & $0.205(0.142)$ \\
\hline reduction & $-0.346(0.385)$ & $-0.012(0.297)$ \\
\hline date & $0.084 * *(0.057)$ & $0.254^{* * *(0.072)}$ \\
\hline number & & $-0.248^{* * *(0.041)}$ \\
\hline rate & & $-1.028^{*}(0.657)$ \\
\hline negp & & $7.546^{* * *(5.821)}$ \\
\hline price & & $0.134^{*}(0.103)$ \\
\hline feature & & $-1.725^{*}(0.826)$ \\
\hline emotion & & $-3.217^{* * *}(1.254)$ \\
\hline$\alpha$ & $3.417 * *(0.546)$ & $4.958^{* * *(0.627)}$ \\
\hline Sample size & 185 & 185 \\
\hline F & $1.24 *$ & $6.02 * * *$ \\
\hline $\mathrm{R}^{2}$ & 0.045 & 0.255 \\
\hline
\end{tabular}

Table 1: The influence from online reviews to quantitative perception of iwom-the cross-section regression analysis

(Note: $*$ represents $\mathrm{p}<0.10 ; * *$ represents $\mathrm{p}<0.05 ; * * *$ represents $\mathrm{p}<0.01$. The Numbers listed in the table are estimates of coefficient for the model, the numbers in brackets are the standard error. The following tables are the same.)

From table 1, we can see that when model 1 only has the control variables without the variables of online reviews, it is only marginal significant $(\mathrm{F}=1.24, \mathrm{P}=0.07)$ and the goodness of fit is poor at the same time $\left(\mathrm{R}^{2}=0.045\right)$. After we added the variables of online reviews to the model 2, the goodness of fit rises from 0.045 to 0.255 . The significance level of model 2 also improves significantly $(\mathrm{F}=6.02)$. Through the results of the cross-section regression analysis of model 1 and 2, we can find that the goodness of fit and significance of model improve significantly. It means that online reviews have important influence on quantitative perception of iwom.

\subsection{The first order difference analysis}

In the cross-section regression analysis, the model 2 regards the variables of online reviews as exogenous variables and finds that the number, rate, feature and emotion of online reviews have positive significant influence on quantitative perception of iwom and the negp of online reviews has negative significant influence on quantitative perception of iwom, while the variables of online reviews are likely to be endogenous variables. Ignoring the influence of endogenous may lead to the estimation results of the variables of online reviews produce errors.

In the regression analysis, introducing endogenous thought is an effective solution to control the error of regression. But in the existing researches, the scholars always regard each attribute variables of online reviews as exogenous variables. The main reason is that the factors which influence the quantitative perception of iwom may not be online reviews, but the unquantifiable internal factors inside the product. Such as the product quality, popularity and so on that mentioned in the analysis of influence factors of file sharing by Oberholzer-Gee [12]. 
In the regression model of the paper, controlling more factors is a practical way to deal with the problem of potential endogeneity. Therefore, the paper establishes the model of cross-section analysis with the control variables of rank, competitor, reduction and date to weaken the influence of the endogenous. But there are many influence factors which are difficult to observation or measurement, they also relate to the online reviews and quantitative perception of iwom. So it is likely to lead to endogenous problems. Therefore, the paper not only weakens the influence of the endogenous by adding the controllable control variable, but also needs to further test whether the endogenous problems still exist. We add the fixed effect $\lambda$ of unquantifiable variables on behalf of the product quality and so on into the model 1and 2 . The test result of fixed effect shows that $\lambda$ is significant different to 0 . It means we miss the important relevant variables in the cross-section regression analysis which leads to overvalue the effect of online reviews. The paper uses the model of first order difference analysis to eliminate the potential effect of endogenous.

We add the fixed effect $\lambda$ of immeasurable variables into the model $2, \lambda$ includes all influence factors of difficult to observation or measurement which influence to the quantitative perception of iwom and can relate to any independent variables. The sample data is divided into two groups by the time, the first set of data uses $t=1$ as the time point, and the second set of data uses $t=2$ as the time point. Keep the two groups of sample data contain the same 103 kinds of mobile phones. Through the first order difference analysis, the fixed effect $\lambda$ can be eliminated. Then we build model with $\mathrm{t}=1$ and $\mathrm{t}=2$ respectively.

Where the definitions of variables (rank, competitor, reduction, date, number, rate, negp, price, feature and emotion) are as same as the formula (1) and (2). $T$ is a dummy variable when $\mathrm{t}=1$, $T=0$, when $\mathrm{t}=2, T=1$, it uses to control the effects by time change. $\alpha_{0}$ is the coefficient of dummy variable. We get the model 3 by the model when $\mathrm{t}=2$ minus the model when $\mathrm{t}=1$.

Model 3:

$$
\begin{aligned}
& \Delta \ln \left(\text { sale }_{i}\right)=\Delta \alpha+\Delta \alpha_{1} \ln (\text { rank })+\Delta \alpha_{2} \ln (\text { competitor })+\Delta \alpha_{3} \text { reduction } \\
& +\Delta \alpha_{4} \ln (\text { date })+\Delta \beta_{1} \ln (\text { number })+\Delta \beta_{2} \frac{1}{\text { number }_{i}}+\Delta \beta_{3} \text { negp } \\
& +\Delta \beta_{4} \ln (\text { price })+\Delta \beta_{5} \frac{1}{\text { number }_{i}} \sum \text { feature }+\Delta \beta_{6} \frac{1}{\text { number }_{i}} \sum \text { emotion }+\alpha_{0}
\end{aligned}
$$

Where the definitions of variables (rank, competitor, reduction, date, number, rate, negp, price, feature and emotion) are as same as the formula (1) and (2). $\Delta$ expresses the change of each variable from $t=1$ to $t=2$. Through the first order difference, the immeasurable variables are eliminated; it means that the errors which caused by the influence factors of difficult to observation or measurement are eliminated. In the model 3 , the dependent variable of $\Delta \ln \left(\right.$ sale $\left._{i}\right)$ expresses the change of the sales rank from two time nodes. The control variables and the independent variables are in a similar way. And we focus on the regression analysis results of the coefficient $\beta_{1}, \beta_{2}, \beta_{3}, \beta_{4}, \beta_{5}$ and $\beta_{6}$ of each variable of online reviews. The results of the first order difference analysis about model 3 are shown as table 2 . 


\begin{tabular}{|l|l|l|}
\hline Variables & Model 2 & Model 3 \\
\hline rank & $0.384^{* *}(0.233)$ & $0.242^{*}(0.153)$ \\
\hline competitor & $0.205(0.142)$ & $-0.143(0.107)$ \\
\hline reduction & $-0.012(0.297)$ & $-0.253(0.427)$ \\
\hline date & $0.254^{* * *(0.072)}$ & $1.423^{* * *}(0.284)$ \\
\hline number & $-0.248^{* * *}(0.041)$ & $-0.515^{* *}(0.157)$ \\
\hline rate & $-1.028^{*}(0.657)$ & $-2.457(1.472)$ \\
\hline negp & $7.546^{* * *}(5.821)$ & $4.285^{* *}(3.024)$ \\
\hline price & $0.134^{*}(0.103)$ & $-0.215^{*}(0.174)$ \\
\hline feature & $-1.725^{*}(0.826)$ & $-0.642^{* * *}(0.467)$ \\
\hline emotion & $-3.217 * * *(1.254)$ & $-4.725^{* * *}(1.851)$ \\
\hline$\alpha_{0}$ & $4.958^{* * *}(0.627)$ & $0.417(0.248)$ \\
\hline Sample size & 185 & 103 \\
\hline $\mathrm{F}$ & $6.02 * * *$ & $3.25^{* * *}$ \\
\hline $\mathrm{R}^{2}$ & 0.255 & 0.178 \\
\hline
\end{tabular}

Table 2: The influence from online reviews to quantitative perception of iwom- the first order difference analysis

The regression results show that the model 3 has a good goodness of fit. Relative to the crosssection analysis, the first order difference analysis has eliminated the fixed effect $\lambda$ of immeasurable variables, thus the influence of influence factors of difficult to observation or measurement is controlled. Therefore, through the first order difference analysis, we can get more accurate estimated results of the influence form online reviews to quantitative perception of iwom.

In the regression analysis of the first order difference, we focus on the changes of estimated results of each variable of online reviews. Combining with the results of the cross section analysis and first order difference analysis, we can know that the real number of online reviews has bigger influence to quantitative perception of iwom than the rate of online reviews. While in the masses of online reviews, the online reviews which contain consumers' positive emotional information are the key reviews which can strengthen quantitative perception of iwom of product.

\section{Conclusions And Implication}

The paper analyzes the influence of each attribute of online reviews on quantitative perception of iwom through the empirical research of relationship between online reviews and quantitative perception of iwom of mobile phones.

Through the analysis of previous research achievements and combining with the particularity of mobile phone, the paper summarizes the key quantifiable factors and unquantifiable factors which have influence on quantitative perception of iwom, including the quantifiable indicators: the number of online reviews, rating, the proportion of negative reviews, the price of the product, and the unquantifiable indicators: the feature words of product attributes, emotional intensity of reviews. In the cross-section regression analysis, the paper regards online reviews as exogenous variable, the results of regression show that the number of online reviews, rating, the feature words of product attributes and emotional intensity of reviews have a positive influence on quantitative perception of iwom, while the proportion of negative reviews and the price of the product have a negative 
influence on quantitative perception of iwom. And the negative influence caused by negative reviews is much more significant than the positive influence caused by positive reviews. But if we regard online reviews as exogenous variable, it may lead to the errors of the results of regression analysis.

Therefore, the paper regards online reviews as endogenous variable, introducing the influence factors of difficult to observation or measurement such as product quality to do regression analysis of the first order difference. The results show that when the influence of potential endogenous is controlled, the number of online reviews and emotional intensity of reviews still have a significant positive influence on quantitative perception of iwom. The proportion of negative reviews still has a significant negative influence on quantitative perception of iwom. The positive influence of the feature words of product attributes is strengthened. But the adjustment form price of product to quantitative perception of iwom changes from negative influence to positive influence, it means that when consumers consider the product quality and other attributes, they more believe that the higher the price, the better the quality. The influence of the rate to quantitative perception of iwom changes from significant to below the level of significance; it means that consumers care more about the essence of content about online reviews rather than the rate of online reviews in the field of mobile phone.

\section{References}

[1] E. J. Lee, S. Y. Shin. When do consumers buy online product reviews Effects of review quality, product type, and reviewer's photo [J]. Computers in Human Behavior. 31(1):356-366(2014)

[2] J. R. Fu, P. H. Ju , C. W. Hsu. Understanding Why Consumers eEngage in Electronic Word-of-mouth Communication: Perspectives from Theory of Planned Behavior and Justice Theory [J]. Electronic Commerce Research \& Applications.14(6):616-630(2015)

[3] A. H. Huang, K. Chen, D. C. Yen, et al. A study of factors that contribute to online review helpfulness [J]. Computers in Human Behavior.48(C):17-27(2015)

[4] C. N. Dellarocas, F. N. Awad, X. Zhang. Exploring the Value of Online Product Reviews in Forecasting Sales: The Case of Motion Pictures [J]. Journal of Interactive Marketing. 21(4): 2345(2007)

[5] W. Duan, B. Gu, A.B. Whinston. Do Online Reviews Matter-An Empirical Investigation of Panel Data [J]. Decision Support System. 45(4):1007-1016(2008)

[6] W. Xu, J. Sun, J. Ma, et al. A Personalized Information Recommendation System for R\&D Project Opportunity Finding in Big Data Contexts [J]. Journal of Network and Computer Applications. 59(1): 362-369(2016)

[7] Y. Liu. Word-of-mouth for Movies: Its Dynamics and Impact on Box Office Receipts [J]. Journal of Marketing. 70:74-89(2006)

[8] R. Ladhari, M. Michaud. EWOM Effects on Hotel Booking Intentions, Attitudes, Trust, and Website Perceptions [J]. International Journal of Hospitality Management. 46(3):36-45(2015) 
[9] C. Lymperopoulos, I. E. Chaniotakis. Price Satisfaction and Personnel Efficiency as Antecedents of Overall Satisfaction from Consumer Credit Products and Positive Word-of-mouth [J]. Journal of Financial Services Marketing. 13:63-71(2008)

[10] J. M. Wooldridge. Econometric Analysis of Cross Section and Panel Data [M]. Cambridge, Mass: The MIT press. (2002)

[11] A. Qazi, K. B. S. Syed, R. G. Raj, et al. A concept-level approach to the analysis of online review helpfulness [J]. Computers in Human Behavior. 58:75-81(2016)

[12] G. F. Oberholzer, K. Strumpf. The Effect of File Sharing on Record Sales: An Empirical Analysis [J]. Journal of Political Economy. 115(1): 1-42(2007) 